\title{
The LZ Dark Matter experiment
}

\author{
W. Lorenzon*广 \\ Randall Laboratory of Physics, University of Michigan, Ann Arbor, Michigan 48109-1040, USA \\ E-mail: lorenzon@umich.edu
}

The LUX-ZEPLIN (LZ) experiment is the most advanced next-generation direct detection experiment under construction to search for dark matter in the Universe. It contains a dual-phase liquid xenon time projection chamber with a total active mass of 7 tons. LZ is implementing various low background techniques to significantly reduce radioactive background and reach an unprecedented level of sensitivity to spin-independent WIMPs. For a WIMP mass of $40 \mathrm{GeV}$, a sensitivity of $2.3 \times 10^{-48} \mathrm{~cm}^{2}$ is expected in 1,000 days of operation. LZ will be located at the Sanford Underground Research Facility (SURF) in South Dakota, at the same location as the recently completed LUX experiment. In this presentation, an overview of the experimental techniques and science reach will be presented.

The European Physical Society Conference on High Energy Physics

5-12 July, 2017

Venice

\footnotetext{
* Speaker.

${ }^{\dagger}$ representing the LZ collaboration
} 


\section{Introduction}

Overwhelming evidence from astrophysical observations indicates that only $20 \%$ of the matter in the universe consists of ordinary matter, while the remaining $80 \%$ is made of some form of matter called "dark matter". Determining the exact nature of this dark matter has emerged as one of the deepest scientific enquiries of our time spanning the fields of cosmology, astrophysics and fundamental particle physics. Despite overwhelming indirect evidence for its existence, dark matter has eluded direct detection. There is good reason to believe that we are now poised at the threshold of discovery, that the next generation of direct detection experiments will not only observe the dark matter directly but will constrain its properties. A particularly well-motivated hypothesis is that the dark matter consists of Weakly Interacting Massive Particles (WIMPs) produced in the hot environment of the early universe and stabilized against further decay by some discrete symmetry. Such particles would interact weakly with ordinary matter and could be detected with a suitably sensitive detector operating in an extremely low background environment. The search for WIMPs is in full swing worldwide using a variety of experimental techniques.

\section{LZ Experiment}

The LZ dark matter experiment, the most advanced next-generation direct detection experiment under construction, will be located in the Davis underground laboratory at the Sanford Underground Research Facility (SURF) in South Dakota, at the same location as the recently completed LUX experiment [1]. It will contain a dual phase (liquid and gas) xenon time projection chamber (TPC) with a total active mass of 7 tons of active liquid Xe (LXe) to search for nuclear recoils from WIMPs scattering off xenon nuclei (for details, see Ref. [2]). The LZ time projection chamber will detect both prompt scintillation light (S1) and proportional scintillation light (S2). Both signals are measured by arrays of photomultiplier tubes (PMTs) above and below the active volume consisting of 247 top and 241 bottom 3-inch-diameter Hamamatsu R11410-20 PMTs with a demonstrated low level of radioactive contamination and high quantum efficiency. The $X Y$ position of an event is determined from the distribution of the S2 signal in the top PMT array, while the $Z$ position is determined from the time delay between the $\mathrm{S} 1$ and $\mathrm{S} 2$ signals. LZ will employ an array of liquid nitrogen $\left(\mathrm{LN}_{2}\right)$-cooled thermosyphons to control the detector temperature and minimize thermal gradients. The purification system is a scaled-up version of the LUX room-temperature gas-phase purification system, and will exploit the liquid/gas heat-exchanger technology developed for LUX [3] to minimize $\mathrm{LN}_{2}$ consumption.

A 3-D model of the LZ detector, situated inside a large water tank is shown in Fig. 1. The water tank is located at the 4,850-foot level of the SURF underground facility. The heart of the LZ detector, including the inner titanium cryostat, will be assembled in a dedicated clean room at SURF that includes air handling to remove radon, lowered in the Yates shaft to the 4,850-foot level, and deployed in the existing water tank in the Davis Cavern. All other components will be staged at SURF, lowered via the Yates shaft, and moved to the cavern. These include the segmented acrylic vessels for the outer detector system and other large components.

LZ entails a fiftyfold scale-up in fiducial mass from LUX [1] (and about a 1,000-fold scaleup from the ZEPLIN [4] target). Its design is enhanced by several added capabilities beyond the 
successfully demonstrated LUX and ZEPLIN designs. The most important addition is a hermetic liquid-organic-scintillator outer detector, which surrounds the central cryostat vessels and the TPC. The outer detector, consisting of 21.5 tons of gadolinium-loaded linear alkylbenzene liquid scintillator material, and the active Xe "skin" layer, which is optically separated from the TPC and is instrumented with 180 PMTs, operate as an integrated veto system. This has the benefit of rejecting internally generated gamma-rays and neutrons that scatter only once in the active region and then escape, thus potentially mimicking a WIMP signal. Since these internally generated background events interact primarily in the outer regions of the detector, the veto allows an increase in the fiducial volume.

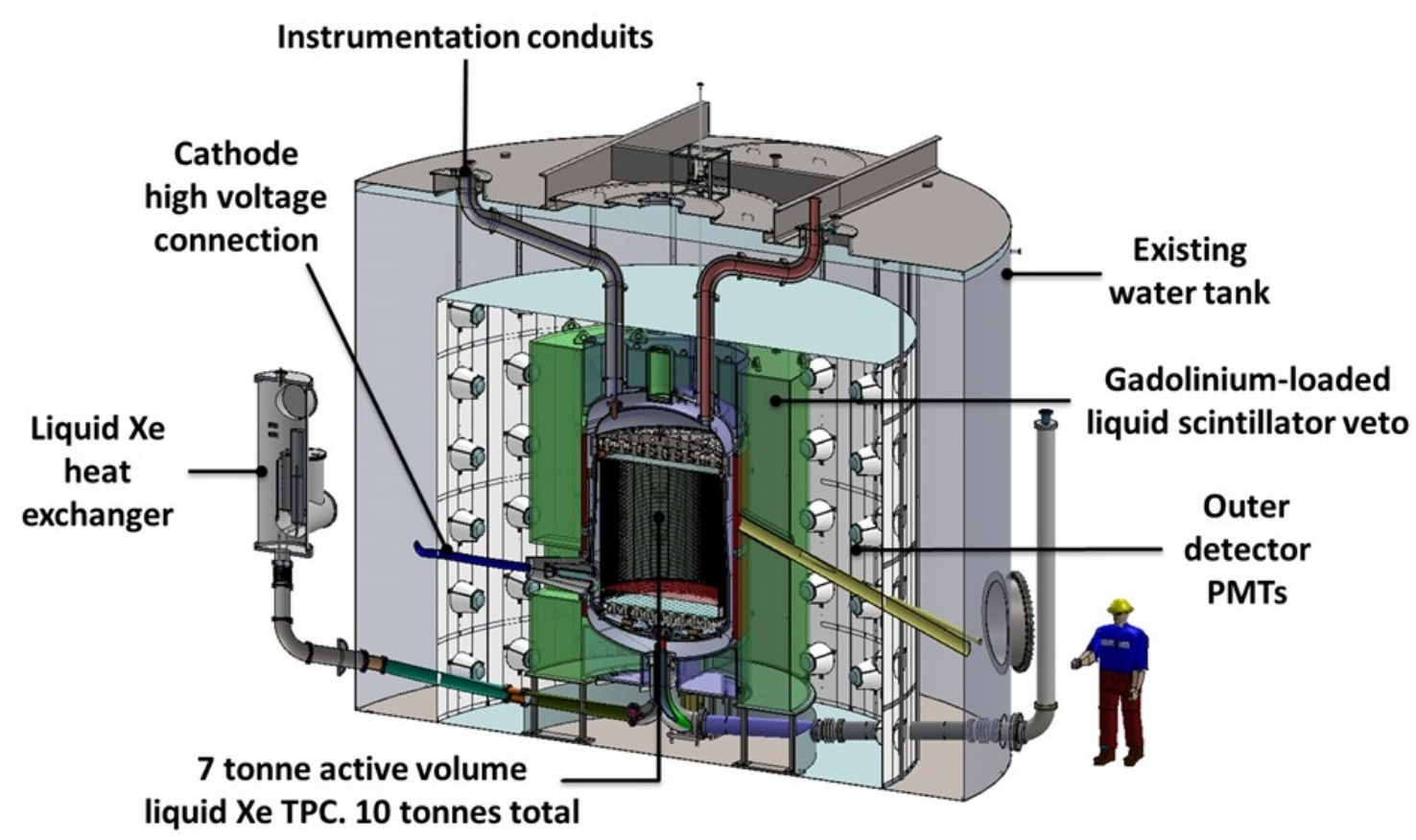

Figure 1: 3-D engineering model of the LZ detector, including water and scintillator shields and central cold Xe volume.

For detector calibration, neutron and gamma-ray sources will be transported to the inner cryostat wall through an array of tubes that penetrate the water and organic scintillator volumes. The principal calibrations, metastable krypton, metastable xenon and tritiated methane, will be introduced directly into the LXe via the xenon gas handling system for in situ electron recoil calibration [5]. An external neutron generator that produces "high"-energy neutrons through a deuteriumdeuterium fusion reaction, as well as additional neutron calibration sources, as for example a Y-Be source for "low"-energy neutrons, will also be employed for nuclear recoil calibration.

\subsection{LZ Science Reach}

Direct searches for dark matter have been pursued for the past thirty years, initially with lowbackground $\mathrm{Ge}$ and $\mathrm{NaI}$ crystals. These did not provide discrimination between electron and nuclear recoils (ER/NR), but with sensitivities reaching $10^{-41} \mathrm{~cm}^{2}$ were capable of ruling out the most straightforward WIMP hypothesis of a heavy Dirac neutrino [6]. This was followed by a decade of 
searches with cryogenic Ge detectors that were able to distinguish NR from ER backgrounds. They achieved sensitivities of $5 \times 10^{-44} \mathrm{~cm}^{2}$ and were able to probe dark matter as Majorana fermions which couple to nucleons via the Higgs mechanism. Over the past decade, noble liquid TPCs have emerged as the most sensitive probes for WIMPs, with the LUX experiment [1] having achieved a sensitivity of $1.1 \times 10^{-46} \mathrm{~cm}^{2}$ at a WIMP mass of $50 \mathrm{GeV}$. Scaling up in size from the LUX design, the LZ detector as shown in Fig. 1 is designed to reach a sensitivity of $2.3 \times 10^{-48} \mathrm{~cm}^{2}$ in 1,000 days for a WIMP mass of $40 \mathrm{GeV}$ and a fiducial mass of $5,600 \mathrm{~kg}$ or above. This is a factor 50 improvement over LUX [1]. Achieving such an ambitious goal requires reducing background events in the fiducial volume to unprecedented low levels by employing shielding, and by identifying and discriminating against background events. Figure 2 summarizes the results from recent direct detection experiments, and shows the estimated sensitivity limit for the LZ experiment, as well as expectations from a variety of SUSY models [7].

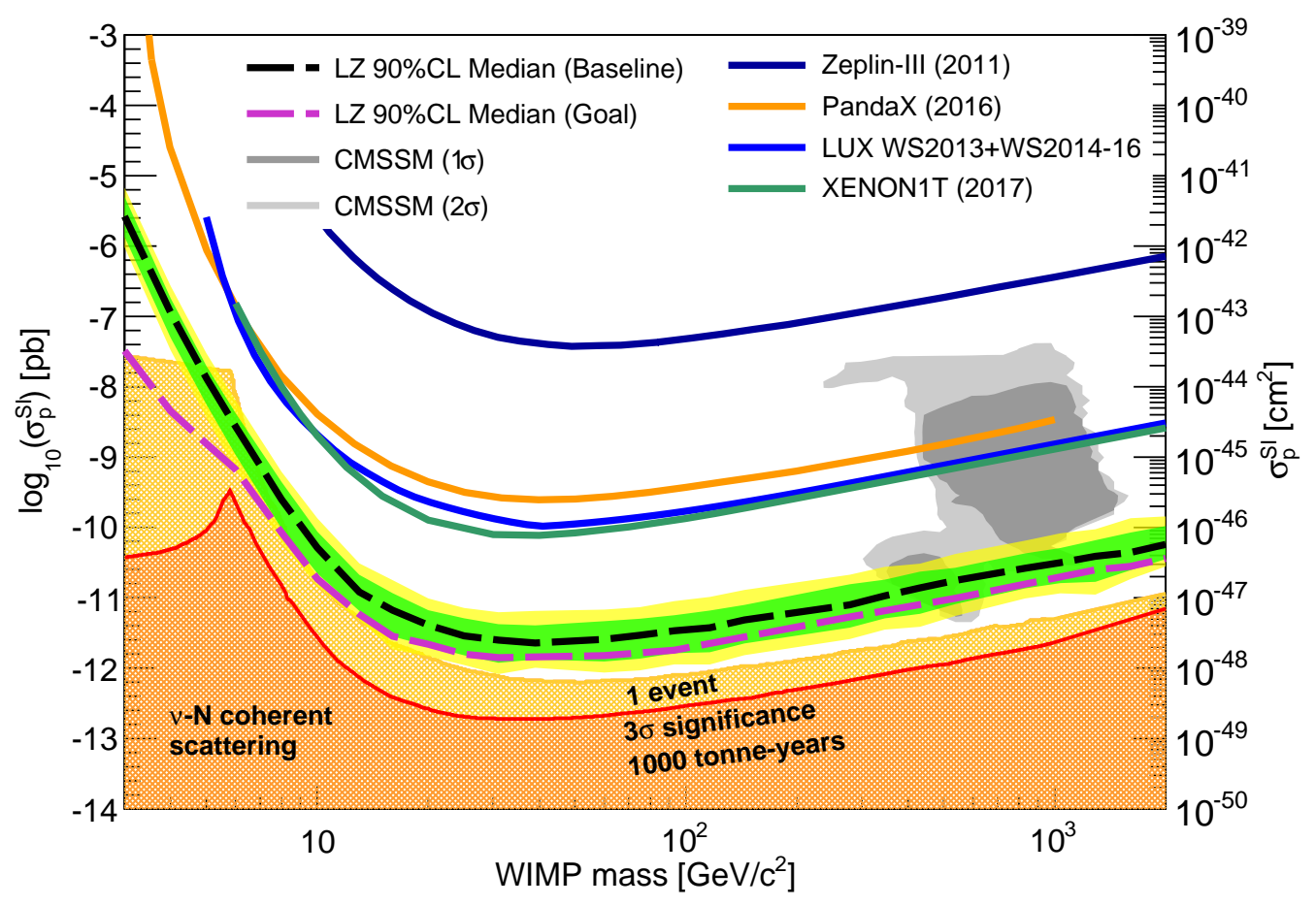

Figure 2: Current spin-independent WIMP-nucleon elastic cross section sensitivity projection (dashed curves) at 90\% C.L. for the LZ experiment. Also shown are results (solid curves) from ZEPLIN-III [9], Xenon100 [10], PandaX [11] and LUX [1], as well as some dark matter models indicated by the shaded regions [7].

LZ will probe the heart of the Higgs scattering region and much of the minimally supersymmetric Standard Model (MSSM) parameter space [7]. WIMP models that will escape the sensitivity of this experiment typically have an exceptional feature (e.g. cancelation in the direct detection amplitudes, finely-tuned parameters, co-annihilation, or a combination of the above) [8]. A null result from this experiment will place strong constraints on the WIMP paradigm. Conversely, this indicates the discovery power of this experiment.

The LZ detector will also be able to perform searches of other rare events such as searches 
for neutrinoless double beta decay (probably at the level of EXO-200 or somewhat better); events from neutrinos from $p p$ fusion in the sun; and events from supernovae in the Milky Way [2]. Note that ERs in the fiducial volume will be dominated by solar neutrinos from $p p$ fusion, which exhibit a flat recoil spectrum in the WIMP search energy region $\left(1.5-6.5 \mathrm{keV}_{e e}\right)$. It is rejection of this signal that drives the performance goal for ER/NR discrimination of $99.5 \%$ or above.

\subsection{Schedule}

The LZ collaboration was formed in March 2012 to perform an ambitious WIMP dark matter program. Initial approval (CD-1/3a and CD-3) was obtained in April 2015 and February 2017, respectively. With LUX removed from underground in March 2017, preparations for surface assembly at SURF began in June 2017. It is currently planned to begin underground installation in July 2018 and commissioning in late 2019, and continue data collection for about 5 years to explore a significant fraction of the available phase space with a WIMP sensitivity of $2.3 \times 10^{-48} \mathrm{~cm}^{2}$ at $40 \mathrm{GeV} / \mathrm{c}^{2}$ with 1,000 live days (and approaching neutrino floor).

\section{References}

[1] D. S. Akerib et al. [LUX collaboration], "Results from a search for dark matter in the complete LUX exposure," Phys. Rev. Lett., 118, 021303 (2017).

[2] B.J. Mount et al. [LZ collaboration], "The LUX-ZEPLIN (LZ) Technical Design Report," arXiv:1703.09144v1 [physics.ins-det].

[3] D. S. Akerib et al. [LUX collaboration], "The LUX prototype detector: Heat exchanger development," Nucl. Instr. Meth. A709, 29 (2013).

[4] D.Yu. Akimov et al. [ZEPLIN collaboration], "The ZEPLIN-III dark matter detector: Instrument design, manufacture and commissioning," Astropart. Phys. 27, 46 (2007).

[5] D.S. Akerib et al. [LUX Collaboration], "First results from the LUX dark matter experiment at the Sanford Underground Research Facility," Phys. Rev. Lett. 112, 091303 (2014).

[6] B.W. Lee and S. Weinberg, "Cosmological Lower Bound on Heavy-Neutrino Masses," Phys. Rev. Lett. 39, 165 (1977).

[7] E. A. Bagnaschi et al., "Supersymmetric Dark Matter after LHC Run 1," Eur. Phys. J. C75, 500 (2015).

[8] T. Cohen, D. J. Phalen, A. Pierce, "On the Correlation Between the Spin-Independent and Spin-Dependent Direct Detection of Dark Matter," Phys. Rev. D81, 116001 (2010).

[9] D.Yu. Akimov et al., "Wimp-nucleon cross-section results from the second science run of ZEPLIN-III." Phys. Lett. B709, 14 (2012).

[10] E. Aprile et al. [XENON collaboration], "Dark Matter Results from 225 Live Days of XENON100 Data." Phys. Rev. Lett. 109, 181301 (2012).

[11] Andi Tan et al. [PandaX collaboration], "Dark Matter Results from First 98.7 Days of Data from the PandaX-II Experiment.” Phys. Rev. Lett. 117, 121303 (2016). 